\begin{tabular}{|c|l|}
\hline Title & Transcription of 4'-thioDNA templates to natural RNA in vitro and in mammalian cells \\
\hline Author(s) & Maruyama, Hideto; Furukawa, Kazuhiro; Kamiya, Hiroyuki; Minakawa, Noriaki; Matsuda, A kira \\
\hline Citation & $\begin{array}{l}\text { Chemical communications, 51(37), 7887-7890 } \\
\text { https://doi.org/40.1039/c4c08862j }\end{array}$ \\
\hline Issue Date & 2015-05-07 \\
\hline Doc URL & http://hdl.handle.net/2115/60827 \\
\hline Type & article (author version) \\
\hline File Information & manuscript.pdf \\
\hline
\end{tabular}

Instructions for use 


\title{
Transcription of 4'-thioDNA templates to natural RNA in vitro and in mammalian cells
}

\author{
Hideto Maruyama, ${ }^{a}$ Kazuhiro Furukawa, ${ }^{b}$ Hiroyuki Kamiya, ${ }^{c}$ Noriaki \\ Minakawa $^{*^{b}}$ and Akira Matsuda ${ }^{*^{a}}$
}

Synthetic chemically modified nucleic acids, which are compatible with DNA/RNA polymerases, have great potential as a genetic material for synthetic biological studies. A comprehensive analysis of the transcription of $4^{\prime}$-thioDNA templates to natural RNA is herein reported. The modifications of DNA with 2'-deoxy-4'-thionucleosides, so-called $4^{\prime}$-thioDNA, were found to act as templates for transcription with RNA polymerases both in vitro and in mammalian cells.

Exploring the compatibility of synthetic chemically modified nucleic acids with DNA/RNA polymerases is an important factor for investigating the evolution of aptamers ${ }^{1}$ and to better understand the information transfer to develop orthogonal genetic systems. ${ }^{2}$ Nucleobase-modified synthetic genetic materials have been explored to expand the genetic code, ${ }^{3}$ to label RNA at a specific position, ${ }^{4}$ and to develop oligonucleotide therapeutics. ${ }^{5}$ Kool's and Romesberg's groups have independently reported the biocompatibility of nucleobase-modified synthetic polymers, which could act as templates for replication with DNA polymerase in E. coli $^{6}$ and transcription with T7 RNA polymerase in vitro. ${ }^{7}$ In addition, phosphodiester backbone-modified ${ }^{8}$ and ribofuranosyl ring-modified ${ }^{9}$ nucleic acids are also expected to have potential as genetic materials. Brown and co-workers reported the transcription of a backbone-modified DNA containing a triazole linkage at a single position instead of a phosphodiester bond to natural RNA in E. coli ${ }^{8 \mathrm{f}}$ and in mammalian cells. ${ }^{8 \mathrm{~g}}$ Because of the high level of nuclease resistance, ribofuranosyl ring-modified nucleic acids may also be a good candidate as a genetic material. ${ }^{9 \mathrm{c}}$ However, there have been few reports investigating the biocompatibility of such polymers due to the negative effect of the helical conformation of the oligonucleotides on their recognition by enzymes. ${ }^{2 b}$

In our previous paper, we showed that 4 '-thioDNAs having a sulfur atom instead of an oxygen atom in the furanose ring could be amplified by PCR in the presence of 2'-deoxy-4'-thio-thymidine and -cytidine 5'-triphosphates (dSTTP and $\mathrm{dSCTP})^{10}$ or even in the presence of all four kinds of 2'-deoxy-4'-thionucleoside 5'-triphosphates (dSNTPs, Fig. 1). ${ }^{11}$ Although the amplified $4^{\prime}$-thioDNAs containing only pyrimidine nucleosides, such as $2^{\prime}$-deoxy-4'-thio-thymidine

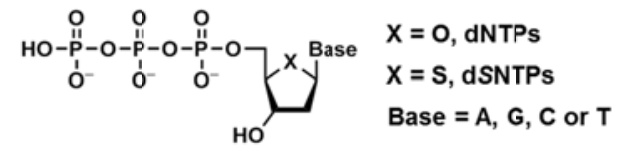

Fig. 1. The chemical structures of $2^{\prime}$-deoxynucleoside 5 -triphosphates (dNTPs) and 2'-deoxy-4'-thionucleoside 5'-triphosphate (dSNTPs).

$(\mathrm{d} S \mathrm{~T})$ and/or -cytidine (dSC) have been proven to act as templates to yield natural RNA in vitro, as well as in mammalian cells, ${ }^{10}$ a comprehensive analysis of the transcription of 4'-thioDNA templates to natural RNA has not yet been performed. In this paper, we report the transcription of 4'-thioDNA templates containing all kinds of 2'-deoxy-4'-thionucleosides (dSNs) including 2'-deoxy-4'-thio-purine nucleosides (dSA and/or dSG) to natural RNA in vitro and in mammalian cells.

First, we evaluated the in vitro transcription of 4'-thioDNA templates (104 mer) prepared by PCR in the presence of various combinations of dSNTPs (as described in the Supporting Information) using T7 RNA polymerase (Figs. 2A, $\mathrm{S} 1)$. As shown in Fig. 2B, the transcription efficiencies when using 4 '-thioDNA templates containing one $\mathrm{d} S \mathrm{~A}, \mathrm{~d} S \mathrm{G}, \mathrm{d} S \mathrm{~T}$ or $\mathrm{d} S \mathrm{C}$ relative to the all-natural template were $86 \%, 120 \%, 37 \%$ and $48 \%$, respectively. We previously reported that the transcription of 4 '-thioDNAs containing $\mathrm{d} S \mathrm{~T}$ and/or $\mathrm{d} S \mathrm{C}$ gave corresponding natural RNA oligomers at a $38 \%-49 \%$ efficiency relative to the natural template. ${ }^{10}$ The results obtained in this work therefore closely agreed with our previous data. ${ }^{10}$ Furthermore, the 4 '-thioDNA template containing both $\mathrm{d} S \mathrm{~A}$ and $\mathrm{d} S \mathrm{G}$ afforded the corresponding RNA more effectively, which was comparable to the efficiency of the natural DNA template.

Encouraged by these outcomes, we carried out a similar experiment using DNA templates with various combinations of $\mathrm{d} S$ Ns. The $4^{\prime}$-thioDNA template containing both $\mathrm{d} S \mathrm{~A}$ and $\mathrm{d} S \mathrm{G}$ acted as the most preferable template for the transcription (relative transcription efficiency $=105 \%$ ). On the other hand, the transcription efficiencies of $4^{\prime}$-thioDNA templates containing $\mathrm{d} S \mathrm{~T}$ and/or dSC were all lower $(6 \%-48 \%)$ than the ones containing the purine counterparts. The fidelity of the transcription from 4 '-thioDNA templates were confirmed by sequencing (as described in the Supporting Information, Fig. $\mathrm{S} 2$ ), and the results showed that the $4^{\prime}$-thioDNA was 
(A) DNA template containing 4'-thio nucleotides

3' -d (ATTATGCTGAGTGATATCCCTGATCGATGCTCACGAGGGACATATGAATGAGCATTCAGCAGCTTGATGGTGCCGGTGAAGRCTGGTGGTCGAATATAAGGCAG) - 5' 5' -d (TAATACGACTCACTATAGGGACTAGCTACGAGTGCTCCCTGTATACT TACTCGTAAGTCGTCGAACTACCACGGCCACTTCAGACCACCAGCTTATATTCCGTC) - 3' T7 promoter
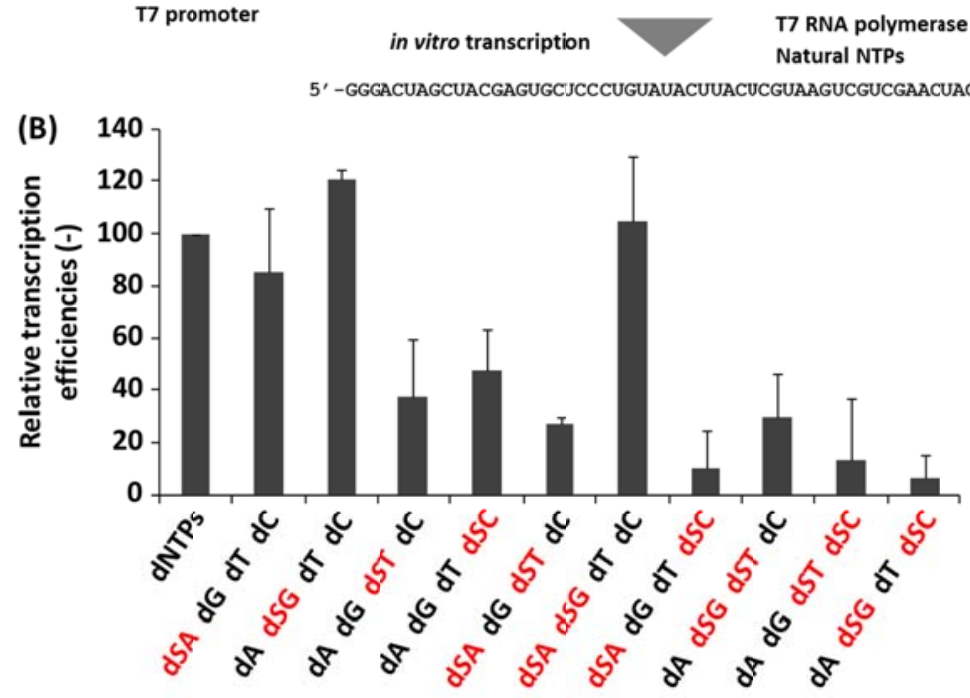

Fig. 2. The in vitro transcription of various DNA templates containing 4 '-thioDNA by T7 RNA polymerase with natural NTPs. (A) The sequence of the DNA template and transcript. DNA templates were prepared by PCR with $\mathrm{d}(S)$ NTPs. The underlined region is the $\mathrm{T} 7$ promoter. (B) The relative transcription efficiencies for each template. The Y-axis indicates the transcriptional yields relative to that frem the natural DNA template. Error bars are the standard deviations of three independent analyses.

transcribed into the natural RNA without losing the sequence information of the template. The transcription with templates containing three or four different $\mathrm{d} S \mathrm{Ns}$ gave almost no transcripts (see Fig. S1). Based on these results, we concluded that the templates containing $\mathrm{d} S \mathrm{~A}$ and/or $\mathrm{d} S \mathrm{G}$ behaved like natural DNA templates, whereas those containing $\mathrm{d} S \mathrm{~T}$ and/or $\mathrm{d} S \mathrm{C}$ were not efficiently recognized as templates by T7 RNA polymerase.

The number of dSTs in the $4^{\prime}$-thioDNA template was 41 in the 104 mer dsDNA, and the numbers of dSCs, dSAs and dSGs were 39,37 and 34, respectively. Therefore, there are no obvious differences in the number of dSNs. In addition, there were no consecutive sequences greater than four for any of the $\mathrm{dSNs}$, although there was one CCC and one GGG sequence in the template. Therefore, the differences in the transcription efficiencies were not due to the numbers of $d S N s$ in the DNA. Although there is a possibility that $\mathrm{d} S \mathrm{~T}$ and/or $\mathrm{d} S \mathrm{C}$ in the template interfered with the interaction with T7 RNA polymerase $^{8 \mathrm{a}}$ further investigations would be needed to elucidate such differences in detail.

To further investigate whether the transcription of 4 '-thioDNA templates containing $\mathrm{d} S \mathrm{~A}$ and/or dSG would give natural RNA transcripts in mammalian cells, the luciferase expression level of plasmids with 4 '-thioDNA modifications was measured for the convenient analysis of the transcription efficiency of 4'-thioDNA (illustrated in Fig. S3). Part of the mRNA coding region in the luciferase expression plasmids was replaced with 4 '-thioDNA prepared by a DNA synthesizer (Fig. 3 ), and the luciferase expression level was compared with that of the unmodified plasmid.

The sequence of the pGL2-control vector $(6.0 \mathrm{kbp})$ was modified (as described in the Supporting Information) to introduce a Bsm BI recognition site and a frame-shift mutation was introduced, which provided a stop codon. The constructed plasmid (pGL2- Bsm I) was treated with Bsm BI and Bst EII (pGL2-Bsm BI/Bsm BI-Bst EII, Fig. S4), and the chemically
pGL2-Bsm BI, antisense strand (834-875)

3' -GTTAACGTGACTATTACTTAAGGAGACCTAGATGACCCAATG-P5'

$$
\int 4 \text { '-thioDNA }
$$

\section{dSAT 3' -GTTAACGIGACTATTACTTAAGGAGACCTAGATGACCCAATG-P5'}

dSGC 3' -GTTAACGTGACTATTACTTAAGGAGACCTAGATGACCCAATG-P5'

dSAG 3' -GTTAACGTGACTATTACTTAAGGAGACCTAGATGACCCAATG-P5'

dSAC 3 ' -GTTAACGIGACTATTACTTAAGGAGACCTAGATGACCCAATG-P5'

dSGT 3' -GTTAACGIGACTATTACTTAAGGAGACCTAGAIGACCCAATG-P5'

dSCT 3' -GTTAACGTGACTATTACTTAAGGAGACCTAGATGACCCAATG-P5'

Fig. 3. The sequences of the DNAs used to prepare 4'-thioDNA modified plasmids. The 2'-deoxy-4'-thionucleotides (shown in red) were introduced into the antisense strand of plasmids by the standard phosphoramidite method. The letter ' $\mathrm{P}$ ' indicates phosphorylation of the 5 '-end of DNA.

synthesized DNAs (42-nt) with two of four dSN modifications (Fig. 3) were introduced into the antisense strand of the plasmids (Fig. S5). Both ends of the five nucleotides in the inserted DNAs were retained as natural DNA to avoid interfering with the ligation with T4 DNA ligase. The insertion of DNAs was confirmed by the reaction of $5^{\prime}-{ }^{32} \mathrm{P}$-labeled DNAs with pGL2-Bsm BI/Bsm BI-Bst EII (Fig. S6), because the band mobility of pGL2-Bsm BI/Bsm BI-Bst EII was unchanged after the ligation reaction (Fig. S4, compared with lane 1 and lanes 2-8). To determine the reaction efficiency, the prepared plasmids were treated with Bam HI and Bsm BI (Fig. S7). The results showed that the reaction efficiency was only a few percent, which might have been caused by the general properties of the short DNA fragment inserted in the plasmid, because the yield of the fragment with natural DNA inserted was as low as that of the other DNA sequences containing $\mathrm{d} S \mathrm{Ns}$.

The prepared plasmids were transfected into NIH3T3 cells with Lipofectamine 2000 based on the manufacturer's protocol, and the luciferase expression was monitored at 24,48 or $72 \mathrm{~h}$ 


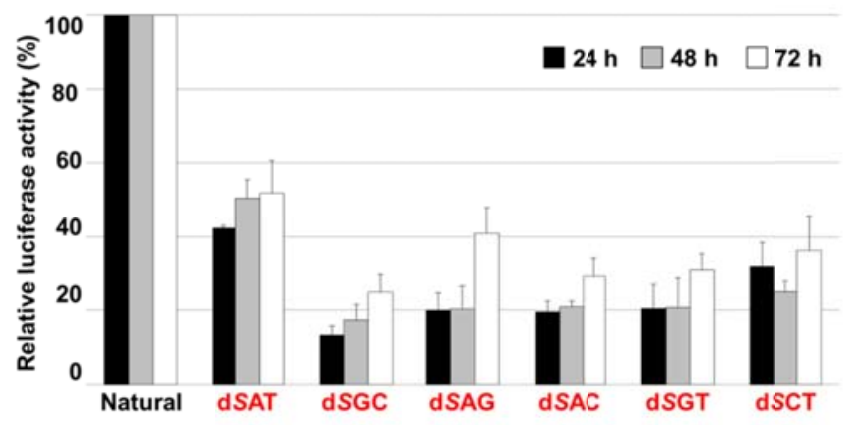

Fig. 4. The transcription efficiencies of the 4'-thioDNA modified plasmid based on the luciferase expression levels. The luciferase expression was monitored at 24,48 or $72 \mathrm{~h}$ post-transfection. The expression of the cells transfected with a plasmid inserted with natural DNA was set at $100 \%$. The plotted data are the means \pm standard deviation of three independent experiments.

post-transfection (Fig. 4). The results showed that all plasmids modified with 4'-thioDNA were transcribed into the corresponding mRNA, and the luciferase protein was translated, although the efficiency was about $20-40 \%$ compared with the plasmid inserted with natural DNA. To check if these less efficiencies were due to the cytotoxicity of $4^{\prime}$-thioDNA, the cells were treated with natural DNA or 4'-thioDNA containing $\mathrm{d} S A G$ and dSAC (Fig. S8). 4'-ThioDNA containing dSAG and $\mathrm{d} S A C$ did not show any cytotoxicity at 24,48 or $72 \mathrm{~h}$ post transfection. In contrast to the in vitro transcription efficiencies, the luciferase expression level of the plasmid modified with 4'-thioDNA containing $\mathrm{d} S \mathrm{~A}$ and $\mathrm{d} S \mathrm{G}$ did not exhibit the highest activity. Because the modifications of DNA with both $\mathrm{dSA}$ and $\mathrm{d} S \mathrm{G}$ resulted in a sequence with seven consecutive 4 '-thioDNA, this might have caused an unfavourable conformational change $^{12}$ for transcription with RNA polymerase. Furthermore, in contrast to single-subunit T7 RNA polymerase, the multi-subunit mammalian RNA polymerase II has a more crowded active site, ${ }^{13}$ which would lead to lower tolerance for a modified nucleotide, although the transcription process of both RNA polymerases is known to occur via a similar mechanism. ${ }^{14}$ In addition, there would be a sequence dependency of DNA to the transcription efficiency, therefore we needed to compare the transcription efficiency of 4'-thioDNAs with different sequences. However it should be mentioned that all four species of dSNs were transcribed into natural mRNAs for up to $72 \mathrm{~h}$ in mammalian cells, demonstrating that the $4^{\prime}$-thioDNA is a promising candidate to serve as a genetic material and also as a bioengineering tool.

In summary, we found the transcription of 4'-thioDNA templates containing $\mathrm{d} S \mathrm{~A}$ and/or $\mathrm{d} S \mathrm{G}$ with T7 RNA polymerase to be as effective as that of the natural DNA. Although, the luciferase expression level of plasmids with 4'-thioDNA modifications showed moderate transcription efficiencies compared with the natural DNA, it was demonstrated that dSA and/or dSG were also transcribed into RNA, similarly to their pyrimidine counterparts. Significantly, there have been no other reports showing that DNA with dozens of sugar-modified nucleotides acted as a template for transcription with RNA polymerase. Therefore, these results, in addition to our previous reports describing the replication of $4^{\prime}$-thioDNA, ${ }^{10-11}$ can thus provide a first step toward the development of synthetic genetic systems ${ }^{2}$ with the synthetic central dogma of $4^{\prime}$-thioDNA/RNA.

\section{Notes and references}

${ }^{a}$ Faculty of Pharmaceutical Sciences, Hokkaido University, Kita-12, Nishi-6, Kita-ku, Sapporo 060-0812, Japan

${ }^{b}$ Graduate School of Pharmaceutical Siciences, The University of Tokushima, Shomachi 1-78-1, Tokushima 770-8505, Japan

${ }^{c}$ Graduate School of Biomedical \& Health Sciences, Hiroshima University, 1-2-3 Kasumi, Minami-ku, Hiroshima 734-8553, Japan

$\dagger$ This work was supported in part by a Grant-in-Aid for Scientific Research from the Japan Society for Promotion of Science (No. 15209003 and 24390027). We are grateful to Dr. Keiji Murakami (The University of Tokushima) for his contributions to the sequence analysis.

Electronic Supplementary Information (ESI) is available: [details of any supplementary information available should be included here]. See DOI: $10.1039 / \mathrm{c} 000000 \mathrm{x} /$

1 (a) J. C. Chaput, H. Yu and S. Zhang, Chem. Biol., 2012, 19, 1360-1371; (b) M. Kimoto, R. Yamashige, K. Matsunaga, S. Yokoyama and I. Hirao, Nat. Biotechnol., 2013, 31, 453-457.

2 (a) D. H. Appella, Curr. Opin. Cherm. Biol., 2009, 13, 687-696; (b) V. B. Pinheiro, D. Loakes and P. Holliger, Bioessays, 2013, 35, 113-122.

3 (a) J. A. Piccirilli, T. Krauch, S. E. Moroney and S. A. Benner, Nature, 1990, 343, 33-37; (b) C. Y. Switzer, S. E. Moroney and S. A. Benner, Biochemistry, 1993, 32, 10489-10496; (c) A. A. Henry and F. E. Romesberg, Curr. Opin. Chem. Biol., 2003, 7, 727-733; (d) I. Hirao and M. Kimoto, Proc. Jpn. Acad., Ser. B, 2012, 88, 345-367.

4 (a) M. Kimoto, M. Endo, T. Mitsui, T. Okuni, I. Hirao and S. Yokoyama, Chem. Biol., 2004, 11, 47-55; (b) R. Kawai, M. Kimoto, S. Ikeda, T. Mitsui, M. Endo, S. Yokoyama and I. Hirao, J. Am. Chem. Soc., 2005, 127, 17286-17295; (c) K. Moriyama, M. Kimoto, T. Mitsui, S. Yokoyama and I. Hirao, Nucleic Aicids Res., 2005, 33, e129; (d) N. Morohashi, M. Kimoto, A. Sato, R. Kawai and I. Hirao, Molecules, 2012, 17, 2855-2876.

5 (a) H. Peacock, E. Fostvedt and P. A. Beal, ACS Chem. Biol., 2010, 5, 1115-1124; (b) H. Peacock, A. Kannan, P. A. Beal and C. J. Burrows, J. Org. Chem., 2011, 76, 7295-7300.

6 (a) A. T. Krueger, L. W. Peterson, J. Chelliserry, D. J. Kleinbaum and E. T. Kool, J. Am. Chem. Soc., 2011, 133, 18447-18451; (b) D. A. Malyshev, K. Dhami, T. Lavergne, T. Chen, N.. Dai, J. M. Foster, I. R. Correa, Jr. and F. E. Romesberg, Nature, 2014, 509, 385-388.

7 S. Ulrich and E. T. Kool, Biochemistry, 2011, 50, 10343-10349.

8 (a) T. Ueda, H. Tohda, N. Chikazumi, F. Eckstein and K. Watanabe, Nucleic Acids Res., 1991, 19, 547-55.2; (b) D. J. King, D. A. Ventura, A. R. Brasier and D. G. Gorenstein, Biochemistry, 1998, 37, 16489-16493; (c) K. Z. He, K. W. Porter, A. Hasan, J. D. Briley and B. R. Shaw, Nucleic Acids Res., 1999, 27, 1788-1794; (d) M. Kuwahara, H. Takeshima, J. Nagashima, S. Minezaki, H. Ozaki and H. Sawai, Biorg. Med. Chem., 2009, 17, 3782-3788; (e) A. H. El-Sagheer and T. Brown, Chem. Commun., 2011, 47, 12057-12058; (f) A. P. Sanzone, A. H. El-Sagheer, T. Brown and A. Tavasisoli, Nucleic Acids Res., 2012, 40, 10567-10575; (g) C. N. Birts, A. P. Sanzone, A. H. El-Sagheer, J. P. Blaydes, T. Brown and A. Tavassolii, Angew. Chem. Int. Ed., 2014, 53, 2362-2365. 
9 (a) S. Pochet, P. A. Kaminski, A. Van Aerschot, P. Herdewijn and P. Marliere, Comptes Rendus Biologies, 2003, 326, 1175-1184; (b) J. J. Chen, X. Cai and J. W. Szostak, J. Am. Chem. Soc., 2009, 131, 2119-2121; (c) V. B. Pinheiro, A. I. Taylor, C. Cozens, M. Abramov, M. Renders, S. Zhang, J. C. Chaput, J. Wengel, S.-Y. Peak-Chew, S. H. McLaughlin, P. Herdewijn and P. Holliger, Science, 2012, 336, 341-344; (d) V. Pezo, F. W. Liu, M. Abramov, M. Froeyen, P. Herdewijn and P. Marliere, Angew. Chem.-Int. Edit., 2013, 52, 8139-8143; (e) V. Pezo, G. Schepers, C. Lambertucci, P. Marliere and P. Herdewijn, Chembiochem, 2014, 15, 2255-2258.

10 N. Inoue, A. Shionoya, N. Minakawa, A. Kawakami, N. Ogawa and A. Matsuda, J. Am. Chem. Soc., 2007, 129, 15424-15425.

11 T. Kojima, K. Furukawa, H. Maruyama, N. Inoue, N. Tarashima, A. Matsuda and N. Minakawa, ACS Synth. Biol., 2013, 2, 529-536.

12 (a) N. Inoue, N. Minakawa and A. Matsuda, Nucleic Acids Res., 2006, 34, 3476-3483; (b) A. Matsugami, T. Ohyama, M. Inada, N. Inoue, N. Minakawa, A. Matsuda and M. Katahira, Nucleic Acids Res., 2008, 36, 1805-1812.

13 A. Dimitri, A. K. Goodenough, F. P. Guengerich, S. Broyde and D. A. Scicchitano, J. Mol. Biol., 2008, 375, 353-366.

14 D. Temiakov, V. Patlan, M. Anikin, W. T. McAllister, S. Yokoyama and D. G. Vassylyev, Cell, 2004, 116, 381-391. 